\title{
Green Synthesis of Silver Nanoparticles by Using Rhizome Extract of Dioscorea oppositifolia L. and their anti microbial activity against Human pathogens.
}

\author{
R.Uma Maheswari ${ }^{1}$, A. Lakshmi Prabha ${ }^{2}$, V. Nandagopalan ${ }^{3}$ and V.Anburaja ${ }^{4}$ \\ ${ }^{1} \& 2$ Department of Plant Science, Bharathidasan University, Tiruchirappalli-24, India \\ ${ }^{3 \& 4}$ PG and Research Department of Botany, National College, Tiruchirappalli-1. India \\ Corresponding author: A. Lakshmi Prabha
}

\begin{abstract}
In the present investigation, synthesis of silver nanoparticles by using rhizome extracts of Dioscorea oppositifolia L. and characterized by using UV spec, FT-IR, DLS and TEM. The extract incubated with $\mathrm{AgNo}_{3}$ showed colour changed of the extract from greenish to reddish brown with intensity increasing during the period of incubation. Hence, the rhizome extract act as a reducing and capping agent. Nanoparticles were characterized by using UV visible absorption spectra has shown the absorbance peak at $409 \mathrm{~nm}$, FT-IR. The synthesized silver nanoparticles were generally found to be effective as antimicrobial agents against some important human pathogens which are prevailing as antibiotic resistance. FT-IR peaks were in the extract ranging from 1000-4000 $\mathrm{cm}^{-1}$ which confirmed the presence of polyphenols with aromatic ring and bound amide region required for the synthesis and stabilization of sliver nanoparticles.DLS shows stability of nanoparticles where as Transmission Electron Microscope confirms the sizewas about $14 \mathrm{~nm}$ and shape of the nanoparticle was found to be spherical in shape.
\end{abstract}

Key words: Dioscorea oppositifolia L, Rhizome, Silver nanoparticles, FTIR, DLS, TEM, Antimicrobial action, Human pathogens.

\section{Introduction}

Nanobiotechnology deals with the synthesis of nanostructures using living organisms. Among the use of living organisms for nanoparticle synthesis, plants have found application particularly in metal nanoparticle synthesis. Use of plants for synthesis of nanoparticles could be advantageous over other environmentally benign biological processes as this eliminates the elaborate process of maintaining cell cultures. Biosynthetic processes for nanoparticles would be more useful if nanoparticles were produced extracellular using plants or their extracts and in a controlled manner according to their size, dispersity and shape. Plant use can also be suitably scaled up for large-scale synthesis of nanoparticles.

Thus, nanoparticles exhibit completely new or improved properties based on specific characteristics such as size, distribution and morphology. Particles in the nanometer size do occur in nature and as a result of industrial processes. The most effectively studied nanoparticles today are those made from noble metals, in particular Ag, Pt, Au, and Pd. Among the above form, silver nanoparticles play a significant role in the field of biology and medicine.

Dioscorea oppositifolia commonly called Chinese yam, cinnamon vine. It is a perennial, fast growing vine in the Dioscoreaceae family. The rhizome has been used as the treatment of poor appetite, chronic diarrhea, asthma, dry coughs, frequent or uncontrollable urination, diabetes, and emotional instability. It contains allantoin, a cell-proliferant that speeds up the healing process. Rhizome juice of this plant can be used to treat snakebites and scorpion stings. Its roots contain the mechanism of silver nanoparticles production was hypothesized that silver ions required the NADPH- dependent nitrate reductase enzyme for their reduction, was secreted by the plants in its extracellular environment. The presence of NADH - dependent nitrate reductase enzyme in extracellular rhizome filterate used for the synthesis of nanoparticles has been confirmed, and the mechanism has been studied [23].

\subsection{Synthesis of silver nanoparticles}

\section{Materials and Methods}

Rhizome of Dioscorea oppositifolia were collected air dried for 10 days. They were ground to a fine powder. $200 \mathrm{mg}$ of both the samples were weighed and mixed separately in $200 \mathrm{ml}$ distilled water in a conical flask. The mixed solutions are heated in a water bath for 10-15 min. A change in colour of the solution was observed during heating processes. The formation of dark brown colour indicates the formation of silver nanoparticles. This colour change is indicated due to the reduction of Ag2+ ions. The silver nanoparticles were separated out by centrifugation and the settled nanoparticles were washed with deionised water. This is taken for further characterization studies. 


\subsection{Characterization of silver nanoparticles}

2.2.1 UV-Vis spectrophotometer analysis

The synthesized silver nanoparticles were first characterized by UV- visible spectrophometer in the range of $250-600 \mathrm{~nm}$ using a quartz cuvette with control as reference. The reduction of siver ions was monitored by measuring the UV-Vis spectrum of the reaction medium at four hours after diluting a small aliquot of the sample into distilled water. The surface Plasmon resonance peaks are found noted to be reliably around $400-450 \mathrm{~nm}$.

\subsubsection{FTIR Analysis}

Further characterization of silver nanoparticles involved Fourier transform infrared spectroscopy by the spectrum in the range $450-4000 \mathrm{~cm}-1$ at revolution of $4 \mathrm{~cm}-1$.To remove any biomass residue or compound that is not the capping ligand of the nanoparticles, the residual solution of $100 \mathrm{ml}$ after reaction was centrifuged at $10,000 \mathrm{rpm}$ for $10 \mathrm{~min}$ and the resulting suspension was dispersed in sterile distilled water. The centrifuging and redispersing process was repeated three times. Finally the nanoparticles are analysed by FTIR.

\subsubsection{TEM Analysis:}

Size and shape distribution of silver nanoparticles were Characterized by transmission electron microscopic (TEM) study a few drops of silver nanoparticle solution were dropped onto a TEM grid, and the residue was removed by a filter paper beneath the TEM grid.

\subsection{Antimicrobial Assays:}

Synthesized silver nanoparticles were tested for antimicrobial activity using agar gel diffusion method against pathogenic bacteria, E.coli, S.typhi, Klebsiella pneumonia, Pseudomonas aerogenosa, Bacillus cereus, E.faecolis. The microbial test organisms were grown in nutrient broth at $37^{\circ} \mathrm{C}$ for 24 hours. A $500 \mu 1$ aliquot of each organism were spread on agar using a cotton swab and allowed to dry for 10minutes. Paper discs loaded with $50 \mu \mathrm{g} / \mathrm{ml} \mathrm{AgNP}$ and reference drugs were placed on the surface of each cultured plate and incubated at $37^{\circ} \mathrm{C}$ for 24 hours after which inhibition zone were measured.

\section{Results and Discussion}

Silver nanoparticles formations from silver ions show an absorption peak around $409.85 \mathrm{~nm}$. FTIR spectrum of the nanoparticles obtained in the present study is presented in Fig .2. Among them the absorption bands are observed in the range of $600-4000 \mathrm{~cm}^{-1}$ is $681.41,1639.03,2072.06$, and $3412.65 \mathrm{~cm}^{-1}$. The band at 1639.03 has been identified as amide II These peaks corresponding to amide II and amide III aromatic rings. The bands at 2072.06 have been identified as $\mathrm{COOH}$-Acid group. The amide groups and $\mathrm{COOH}$ groups are acts as a ligand for nanoparticle synthesis. At particle sizes below 100 nanometers the optical scattering efficiency drops rapidly with particle size. DLS report gives the average particle size of the nanoparticle is around $100 \mathrm{~nm}$. TEM image showed the size of the nanoparticle is $14 \mathrm{~nm}$ which will be more suitable for drug production.

Using plants for nanoparticle synthesis can be advantageous over other biological processes by eliminating the elaborate process of maintaining cell cultures. It can also be suitably scaled up for large-scale synthesis of nanoparticles. These reasons make the biological synthesis of nanoparticles more valuable, though people are thinking that their use is similar to that gained by chemical methods. This production through plant, however, will give a positive message that nanoparticles synthesized through greener routes are much safer for human use [7]. Nanotechnology is mainly concerned with synthesis of nanoparticles of variable sizes, shapes, chemical compositions and controlled dispersity and their potential use for human benefits. Although chemical and physical methods may successfully produce pure, well-defined nanoparticles, these are quite expensive and potentially dangerous to the environment [2]. The use of biological organisms such as microorganisms, plant extract or plant biomass could be an alternative to chemical and physical methods for the production of nanoparticles in an eco-friendly manner [1-3]. Similarly, several other metal nanoparticles give characteristic absorption peaks. A progressive increase in the characteristic peak with increase in reaction time and concentration of plant extracts with salt ions is a clear indicator of nanoparticle formation [6]. UV-vis absorption spectra show peaks characteristic of the surface plasmon resonance of nanosized particles [4, 13,17].The silver nanoparticles were found to possess potent antibacterial activity against both Gram-negative and Gram-positive bacteria. Beta-lactam (piperacillin) and macrolide (erythromycin) antibiotics showed a 3.6-fold and 3-fold increase, respectively, in combination with silver nanoparticles selectively against multidrug-resistant Acinetobacter baumannii [16]. Metal nanoparticles such as silver have free electrons, which give rise to SPR absorption band. The characteristic SPR resonance band of biogenic AgNPs occurred at 400-450nm for reaction 
carried out at microwave and room temperature, respectively. The reactants are consumed rapidly eventually leading to the formation of smaller nanoparticles.

. The biologically synthesized silver nanoparticles using medicinal plants were found to be highly toxic against different pathogenic bacteria. The silver nanoparticles of Dioscorea oppositifolia L. shows highest antibacterial activity was observed against E.coli, Klebsiella pneumonia Pseudomonas auerogenosa, Bacillus cereus, S.typhi, E.faecalis.

\section{Conclusion:}

Plants or their extracts can be efficiently used in the synthesis of silver nanoparticles as a greener route. Control over the shape and size of nanoparticles seems to be very easy with the use of plants._In the present study we found that rhizome can be also good source for synthesis of silver nanoparticles. This approach toward the synthesis of silver nanoparticles has many advantages such as, ease with which the process can be scaled up, economic viability, etc. Applications of such eco-friendly nanoparticles in bactericidal, wound healing and other medical and electronic applications, makes this method potentially exciting for the large-scale synthesis of other inorganic materials (nanomaterials).This is the first report on the synthesis of silver nanoparticles using D.oppositifolia L. Rhizome extract and their effect as antimicrobial agents. Such nanoparticles produced using plants have been used in various applications for human benefit. However, the mechanism of such nanoparticle synthesis by plants is used in the new way of Drug production for the disease, which is prevailing as an antibiotic resistance [20-22].

Table1:Antibacterial activity of Silvernanoparticles from Rhizome Extract of Dioscorea oppositifolia L. Zones of inhibition against S.typhi, E.coli, Bacillus cereus, Enterococcus faecolis and upon application of reference drug streptromycin and gentamycin.

\begin{tabular}{|l|l|l|l|l|l|}
\hline \multirow{2}{*}{ S.NO } & \multirow{2}{*}{ Bacterial species } & \multicolumn{4}{|c|}{$\begin{array}{c}\text { Zone of inhibition } \\
\text { (Dioscorea sp })\end{array}$} \\
\cline { 4 - 6 } & & \multirow{2}{*}{$\begin{array}{l}\text { Rhizome } \\
\text { extract }\end{array}$} & $\begin{array}{l}\text { SNPs } \\
\text { (rhizome) }\end{array}$ & Reference drug \\
\cline { 3 - 6 } & & & & & \multicolumn{4}{|c|}{ Gentamycin } & Streptomycin \\
\hline 1. & S.typhi & $19 \mathrm{~mm}$ & $10 \mathrm{~mm}$ & $23 \mathrm{~mm}$ & $1 \mathrm{~mm}$ \\
\hline 2. & E.coli & $14 \mathrm{~mm}$ & $11 \mathrm{~mm}$ & $27 \mathrm{~mm}$ & $25 \mathrm{~mm}$ \\
\hline 3. & Bacillus cereus & $21 \mathrm{~mm}$ & $10 \mathrm{~mm}$ & $23 \mathrm{~mm}$ & $2 \mathrm{~mm}$ \\
\hline 4. & E.faecolis & - & $12 \mathrm{~mm}$ & $18 \mathrm{~mm}$ & - \\
\hline 5. & P.aeruginosa & $18 \mathrm{~mm}$ & $13 \mathrm{~mm}$ & - & $10 \mathrm{~mm}$ \\
\hline 6. & K.pneumonia & $14 \mathrm{~mm}$ & $15 \mathrm{~mm}$ & - & $8 \mathrm{~mm}$ \\
\hline
\end{tabular}

Figure 1: UV-Vis Spectrum

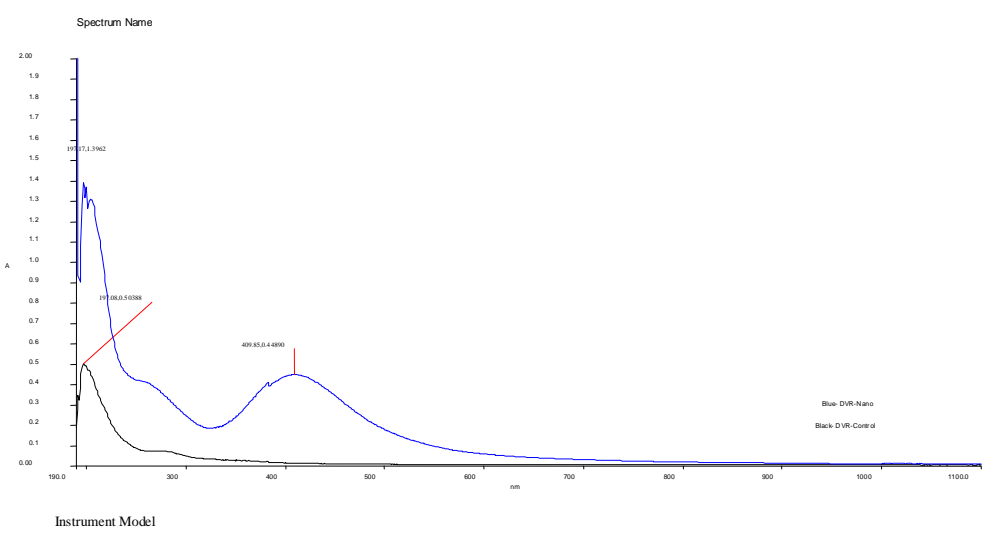


Figure 2: FTIR Spectrum Analysis

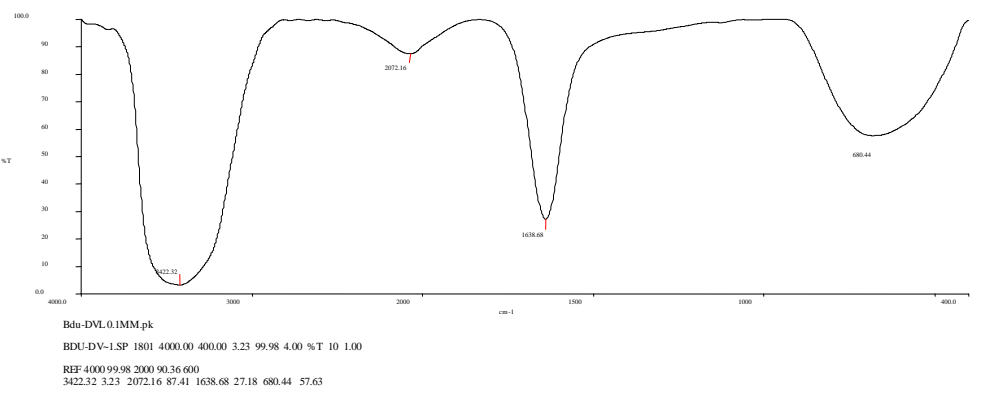

Figure 3: DLS - Dynamic Light Scattering

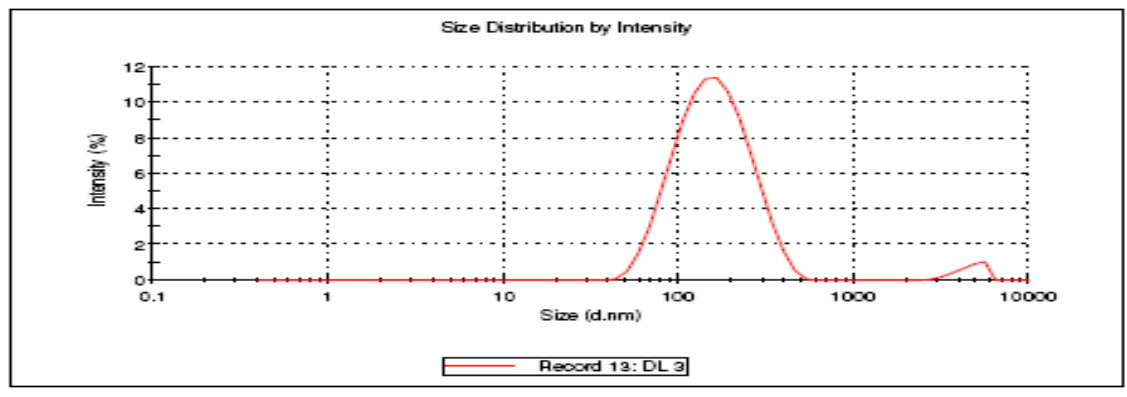

The size of the nano particle is around 100nm, which is confirmed by DLS (Dynamic Light Scattering).

Figure 5: Size of the Nanoparticles produced-14nm.

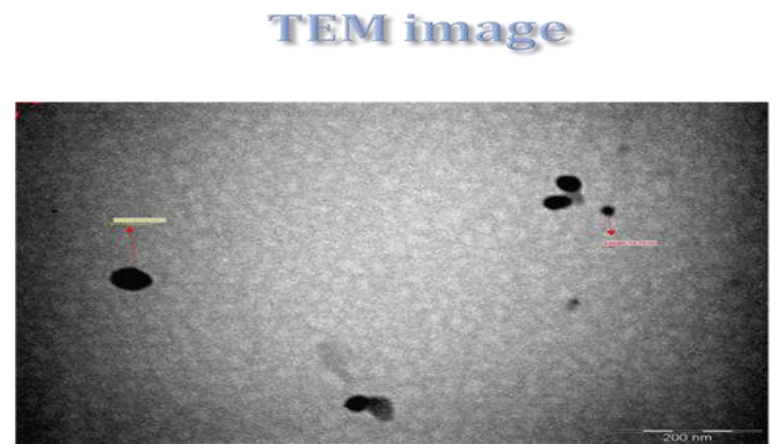

\section{Reference:}

[1]. Ankamwar B, Chaudhary M and Sastry M, Gold nanotriangles biologically synthesized using tamarind leaf extract and potential application in vapor sensing. Synth React Inorg Metal-Org Nano-Metal Chem 35: 19-26 (2005).

[2]. Armendariz V, Gardea-Torresdey JL, Jose-Yacaman M, Gonzalez J, Herrera I and Parsons JG, Gold nanoparticles formation by oat and wheat biomasses, in Proceedings-Waste Research Technology Conference at the Kansas City, Mariott-Country Club Plaza July30-Aug1 (2002).

[3]. Bailey, L.H. and E.Z. Bailey. The MacMillan Company, New York 1949. Hortus Second: A Concise Dictionary of Gardening and General Horticulture.

[4]. Bailey, L.H. The MacMillan Company, New York 1949. Manual of Cultivated Plants.

[5]. Becker RO, Silver ions in the treatment of local infections. Met Based Drugs 6: 297-300 (1999).

[6]. Beyerl, T. Habitat and life history characteristics of Dioscorea oppositifolia, an invasive plant species in southern Illinois. M.S. Thesis, Southern Illinois University, 104pp.( 2001)

[7]. Chandran SP, Chaudhary M, Pasricha R, Ahmad A and Sastry M, Synthesis of gold nanotriangles and silver nanoparticles using Aloe vera plant extract. Biotechnol Prog 22: 577-583 (2006).

[8]. Crumbliss AL, Perine SC, Stonehuerner J, Tubergen KR, Zhao J, Henkens RW, et al, Colloidal gold as a biocompatible immobilization matrix suitable for the fabrication of enzyme electrodes by electrodeposition. Biotechnol Bioeng 40: 483-490 (1992).

[9]. Durán N, Marcato PD, Alves OL, De Souza GIH and Esposito E, Mechanistic aspects of biosynthesis of silver nanoparticles by several Fusarium oxysporum strains. J Nanobiotechnol 3: 8-14 (2005).

[10]. Freeman RG, Grabar KC, Allison KJ, Bright RM, Davis JA, Guthrie AP, et al, Self-assembled metal colloid monolayers: an approach to SERS substrates. Science 267: 1629-1632 (1995). 
[11]. Gardea-Torresdey JL, Gomez E, Peralta-Videa JR, Parsons JG, Troiani H and Jose-Yacaman M, Alfalfa sprouts: a natural source for the synthesis of silver nanoparticles. Langmuir 19: 1357-1361 (2003).

[12]. Gardea-Torresdey JL, Parsons JG, Gomez E, Peralta-Videa J, Troiani HE, Santiago P, et al, Formation and growth of Au nanoparticles inside live alfalfa plants. Am Chem Soc 2: 397-401 (2002).

[13]. Ghule K, Ghule AV, Liu JY and Ling YC, Microscale size triangular gold prisms synthesized using Bengal gram beans (Cicer arietinum L.) Extract and haucl ${ }_{4} \times 3 \mathrm{H}_{2} \mathrm{O}$ : a green biogenic approach. J Nanosci Nanotechnol 6: 3746-3751 (2006).

[14]. Gleason, H.A. and A. Cronquist.). Manual of Vascular Plants of Northeastern United States and Adjacent Canada. The New York Botanical Garden, Bronx. 1991 (1998 update

[15]. Haverkamp RG, Marshall AT and van Agterveld D, Pick your carats: nanoparticles of gold-silver-copper alloy produced in-vivo. J Nanopart Res 9: 697-700 (2007).

[16]. Hilde Jans, Xiong Liu , Lauren Austin, Guido Maes and Qun Hu: Dynamic Light Scattering as a Powerful Tool for Gold Nanoparticle Bioconjugation and Biomolecular Binding Studies._Anal. Chem., 2009, 81 (22), pp 9425-9432.

[17]. Huang J, Li Q, Sun D, Lu Y, Su Y, Yang X, et al, Biosynthesis of silver and gold nanoparticles by novel sundried Cinnamomum camphora leaf. Nanotechnology 18: 105104-105114 (2007).

[18]. Illinois Department of Natural Resources. Chinese Yam Alert! The Natural Areas Association Issues.

[19]. Kumar.V, Yadav.S, Plant-mediated synthesis of silver and gold nanoparticles and their applications, Journal of Chemical Technology and Biotechnology, Volume pages 151-157, February 2009.

[20]. Lópeza ML, Parsonsb JG, Peralta Videab JR and Gardea-Torresdey TL, An XAS study of the binding and reduction of Au (III) by hop biomass. Microchem J 81: 50-56 (2005).

[21]. Mukherjee PK, Saritha GS, Suresh B Antimicrobial potential of two different Hypericum species available in India. Phytother. Res., 16: 692-695(2002).

[22]. Nagajyothi P.C ,Lee K. D, Synthesis of Plant-Mediated Silver Nanoparticles Using Dioscorea batatas Rhizome Extract and Evaluation of Their Antimicrobial Activities. Journal of Nanomaterials Volume 2011, Article ID 573429,7 pages, 8 June 2011.New York)

[23]. TU, M. 2002. Element Stewardship Abstract for Dioscorea oppositifolia L. syn. Dioscorea batatas Decn. Available: http://tncweeds. ucdavis.edu/esadocs/documnts/diosopp.html. The Nature Conservancy, Arlington, VA. 